\title{
Diversidad de las comunidades de peces en dos áreas marinas protegidas del Caribe y su relación con el pez león
}

\author{
Dorka Cobián Rojas ${ }^{1 *}$, Juan J. Schmitter-Soto², Alfonso Aguilar-Perera ${ }^{3}$, \\ Consuelo M. Aguilar Betancourt ${ }^{4}$, Miguel Á. Ruiz-Zárate ${ }^{2}$, Gaspar González Sansón ${ }^{4}$, \\ Pedro P. Chevalier Monteagudo ${ }^{5}$, Alain García Rodríguez ${ }^{6}$, Roberto Herrera Pavón ${ }^{2}$, \\ Susana Perera Valderrama ${ }^{7}$, Hansel Caballero Aragón ${ }^{5}$ \& Elena de la Guardia ${ }^{8}$ \\ 1. Parque Nacional Guanahacabibes, Centro de Investigaciones y Servicios Ambientales (ECOVIDA), La Bajada, \\ Sandino, Pinar del Río, Cuba; dorkacobianrojas79@gmail.com \\ 2. El Colegio de la Frontera Sur, México; jschmitt@ecosur.mx, maruizzar@ecosur.mx, rherrera@ecosur.mx \\ 3. Departamento de Biología Marina, Universidad Autónoma de Yucatán, México; alfaguilar@gmail.com \\ 4. Universidad de Guadalajara, México, Canadian Rivers Institute; coquiaguilar06@yahoo.es, \\ gaspargonzalez2001@yahoo.es \\ 5. Acuario Nacional de Cuba, La Habana, Cuba; pedroc@acuarionacional.cu, hansel@acuarionacional.cu \\ 6. Instituto de Oceanología; alain.garcia2285@gmail.com \\ 7. Centro Nacional de Áreas Protegidas, La Habana, Cuba; susana.perera@gmail.com \\ 8. CINVESTAV- I. P. N. -Unidad Mérida, Yucatán, Ant. Carr. a Progreso Km. 6, A.P. 73 Cordemex, 97310, México; \\ elenadelaguardia@yahoo.com \\ * Correspondence
}

Recibido 13-VI-2017. Corregido 17-VII-2017. Aceptado 03-VIII-2017.

\begin{abstract}
Diversity of native reef fish communities in two protected areas in the Caribbean Sea and its relationship to the invasive lionfish. Lionfish (Pterois volitans) invaded the Caribbean region with the potential to alter the composition and structure of native coral reef fish communities. The objective of this study was to analyze the diversity indices of these fish communities potentially affected by lionfish predation and to compare with pre-invasion data. The study was undertaken in two Caribbean marine protected areas (MPAs): Guanahacabibes National Park (PNG) in W Cuba and Xcalak Reefs National Park (PNAX) in S Quintana Roo. We carried out visual censuses of fish species in reef habitats during the dry and rainy seasons of the period 2013-2015. For this, nine sites were defined and evaluated using stationary counts. Our results showed higher species richness $(43.47 \pm 5.14)$ and mean abundance $(0.76 \pm 1.25)$ in PNG than in PNAX $(40.22 \pm 4.96,0.19$ \pm 0.46 , respectively). Diversity decreased after the arrival of lionfish in a single site of PNG and in two sites of the PNAX, but apparently, these results are more related to the fishing activity effect than to the lionfish presence. Based on the results and assuming that changes in the native fish communities by lionfish may not yet be detected, we recommend to continue the monitoring community descriptions in order to detect future changes in native fish communities. Rev. Biol. Trop. 66(1): 189-203. Epub 2018 March 01.
\end{abstract}

Key words: diversity, invasive species, marine fishes, coral reef, predator effects.

La diversidad es una característica susceptible de ser medida en los ecosistemas marinos, como la productividad, densidad y biomasa (Alcolado, 1998). Los índices de diversidad reflejan la complejidad y estructura del sistema en estudio (Pecenko, 1982), aunque otros autores reconocen que éste es un tema principal a tratar en la ecología (Magurran, 2004;
Rodríguez-Zaragoza et al., 2011). Así como el concepto ha generado discusión semántica, también es polémico el origen de los factores que determinan la diversidad (Alcolado, 1998). Se recomienda utilizarlo como un concepto genérico que englobe los conceptos específicos de riqueza, heterogeneidad, equidad, entre otros índices que se complementen entre sí, 
según los objetivos y diseño de la investigación (Peet, 1974; Rodríguez-Zaragoza et al., 2011).

Actualmente, muchos grupos de gestión y conservación de los ecosistemas utilizan el declive de la diversidad como medio para justificar las decisiones en políticas de manejo (Heller \& Zavaleta, 2009; Rands et al., 2010; Wong, Jiang, Kinzig, Lee, \& Ouyang, 2015). Las naciones caribeñas reconocen cada vez más la riqueza natural de los arrecifes coralinos (Hughes et al., 2003), donde los ecosistemas marinos con mayor diversidad tendrán mejor funcionamiento y resiliencia.

La implementación de áreas marinas protegidas (AMP) se ha convertido en una herramienta efectiva para la conservación de la biodiversidad en los arrecifes coralinos (Gell \& Roberts, 2003; Russ \& Alcala, 2004). Se ha demostrado la efectividad de las AMPs respecto al incremento de la riqueza de especies de peces en arrecifes coralinos (Gell \& Roberts, 2002; Willis, 2003; Palumbi, 2004), también como en el aumento de la resiliencia en comunidades arrecifales (Mellin, MacNeil, Cheal, Emslie, \& Caley, 2016); sin embargo, el sistema global actual de AMP es insuficiente para proteger la diversidad total de corales y peces (Mouillot et al., 2016).

Dentro de las causas de la pérdida de diversidad figuran la destrucción del hábitat, la sobreexplotación, la contaminación, el cambio climático, y las especies invasoras (Santos \& Tellería, 2006; Gutiérrez, 2006; Capote López, Mitrani Arenal, \& Suárez, 2012). Las especies invasoras son aquellas que se establecen en ecosistemas naturales o seminaturales, y constituyen un agente de cambio y de amenaza a la diversidad biológica nativa (Mendoza, Luna, \& Aguilera, 2015). Dentro de los principales impactos ecológicos que provocan destacan la degradación de los hábitats, el desequilibrio ecosistémico, el desplazamiento y la extinción de la flora y fauna nativa, la alteración de la estructura trófica, la facilitación de invasiones subsiguientes y la transmisión de enfermedades (Gutiérrez, 2006).

El pez león (Pterois volitans/miles) es reconocido como la mayor invasión marina en el
Atlántico y el Caribe (Hixon, Green, Albins, Akins, \& Morris, 2016), donde actúa como un competidor muy efectivo, que a largo plazo podría convertirse en una especie dominante que impacta la diversidad de los arrecifes coralinos (Côté, Green, \& Hixon, 2013; Albins, 2015). Estudios experimentales controlados de campo demuestran que el pez león afecta la abundancia local de los peces nativos, lo que puede reflejarse en la diversidad de estos ecosistemas marinos (Green, Akins, Maljković, \& Côté, 2012; Ballew, Bacheler, Kellison, \& Schueller, 2016).

El presente estudio se realizó en dos parques nacionales -Guanahacabibes, Cuba (PNG) y Arrecifes de Xcalak, México (PNAX)- ubicados en el Mar Caribe. Ambos cuentan con información sobre la estructura, composición y diversidad de sus comunidades de peces nativos (Cobián \& Chevalier Monteagudo, 2009; Cobián, Claro, Chevalier, Perera, \& Caballero, 2011; Schmitter-Soto et al., 1998; MoralesAranda, Schmitter-Soto, \& Herrera Pavón, 2012). En el PNG y el PNAX se detectó el pez león desde 2009 (Chevalier et al., 2008; Sabido-Itzá, Gómez Poot, Medina, García Rivas, \& Hadad López, 2012). Monitoreos realizados en ambas áreas demuestran que su población se incrementó de manera significativa (Cobián et al., 2016; SabidoItzá et al., 2012). El objetivo de este estudio fue analizar la diversidad de las comunidades de peces nativos (que pudieran ser afectadas por el pez león) en dos áreas marinas protegidas del Caribe y compararla con datos previos a la invasión del pez león.

\section{MATERIALES Y MÉTODOS}

Zona de estudio: El Parque Nacional Guanahacabibes (PNG) se ubica en la zona más occidental de Cuba con un área total de 39830 ha. La zona de estudio se extendió desde Cabo Corrientes $\left(21^{\circ} 45^{\prime} \mathrm{N}\right.$; 84³0'W) hasta las cercanías del Verraco $\left(21^{\circ} 91^{\prime} \mathrm{N}\right.$, $\left.84^{\circ} 61^{\prime} \mathrm{W}\right)$ (Fig. 1). Los arrecifes estudiados son tipo costero y su perfil característico generalmente es el de una terraza única (González-Ferrer et al., 2007) que culmina en un escarpe profundo de estructura variada y gran 


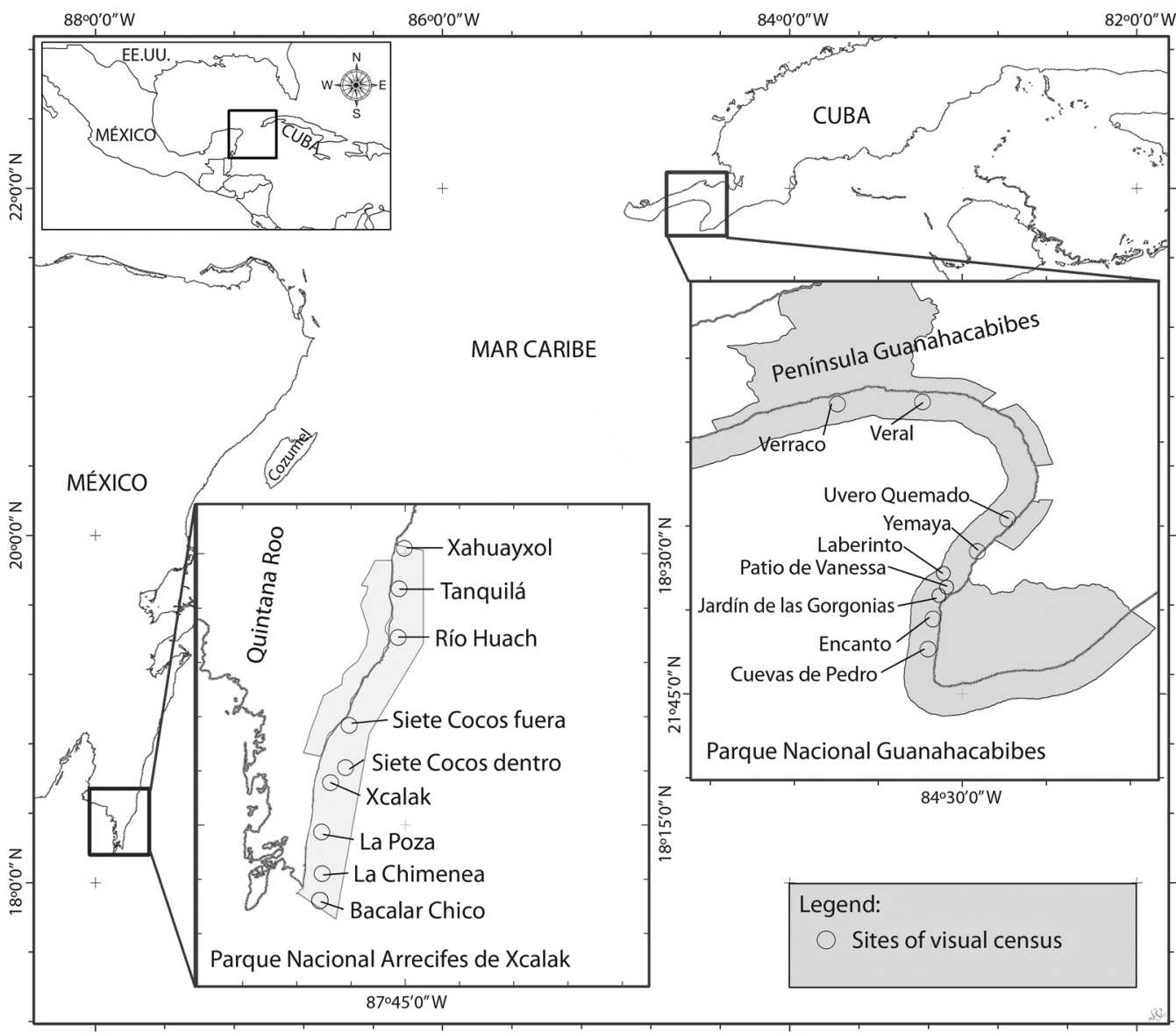

Fig. 1. Sitios de muestreo en el Parque Nacional Guanahacabibes (Cuba) y Parque Nacional Arrecifes Xcalak (México). Fig. 1. Location of sampling sites in Guanahacabibes National Park (Cuba) and Xcalak Reef National Park (Mexico).

arquitectura. El Parque Nacional Arrecifes de Xcalak (PNAX), en México, abarca una superficie total de 17949456 ha, ubicado al sur de Quintana Roo. El área de muestreo se extendió desde Xahuayxol $\left(18^{\circ} 30^{\prime} \mathrm{N} ; 87^{\circ} 45^{\prime} \mathrm{W}\right)$ hasta Bacalar chico $\left(18^{\circ} 12^{\prime} \mathrm{N}\right.$; 8749' W) (Fig. 1). Está área presenta una barrera arrecifal semicontinua a lo largo de la costa, que divide la laguna arrecifal (dominada por pastos marinos y parches aislados de corales escleractinios) del frente arrecifal (elevada diversidad y densidad de corales y gorgonias) (Núñez-Lara, González-Salas, Ruiz-Zárate, Hernández-Landa, \& Arias-González, 2003).
Comunidades de peces: Se realizaron conteos visuales estacionarios (Bohnsack \& Bannerot, 1986) en nueve sitios del PNG, y otros nueve del PNAX (Fig. 1), durante las épocas de secas (PNG: febrero 2014 y 2015; PNAX: abril 2014 y 2015) y lluvias (PNG: junio 2013 y 2014; PNAX: septiembre 2014 y 2015). Ambos arrecifes fueron muestreados cuatro veces, y en cada campaña se realizaron 20 censos por sitio. En cada unidad de muestreo se contabilizó el número total de individuos por especie, dentro de un cilindro imaginario de $5 \mathrm{~m}$ de radio con el observador en el centro, rotando $360^{\circ}$ durante cinco 
minutos. Los datos fueron registrados por dos ictiólogos de experiencia. En el PNG se trabajó en el arrecife frontal entre $12-15 \mathrm{~m}$ de profundidad (cuatro sitios umbral, tres sitios veril, dos sitios camellones y cangilones), mientras que en el PNAX se muestreó en el arrecife frontal en la misma profundidad que en Cuba (cinco sitios camellones y cangilones, dos sitios en el borde de la pared arrecifal y un sitio grieta) y en el interior de la laguna arrecifal (3-5 m) (un sitio: cabezos y parches).

Antes-después del pez león: En Cuba, se tienen registros de las comunidades de peces de cuatro sitios (Cuevas de Pedro, Encanto, Yemayá y Jardín de las Gorgonias) de los años 2003, 2004, 2007, 2008 y 2010, y en México de tres sitios (Xahuayxol: 1997, 1998, 2006, 2010; Río Huach: 1997, 1998, 2006; y Xcalak: 2006, 2007, 2010). Para la comparación, se realizaron muestreos en los mismos sitios teniendo en cuenta los métodos empleados históricamente. En el PNG se ejecutaron censos visuales en seis transectos lineales de $50 \mathrm{~m}$ de largo por $2 \mathrm{~m}$ de ancho (Brock, 1954) desde el 2003-2015. En el PNAX se realizaron 20 censos estacionarios (Bohnsack \& Bannerot, 1986) en cada sitio en los años 2014-15, que se compararon con 10 censos realizados en Rio Huach y 27 censos en Xcalak en 2006-2007. En caso de esfuerzo desigual, los conteos en cada sitio fueron estandarizados por rarefacción (Colwell, 2009) para poder establecer comparaciones.

A partir de la abundancia total, en cada sitio se calculó el índice de riqueza de Margalef (D'n) (Margalef, 1951) definido como: $\mathrm{D}^{\prime}=\mathrm{S}-1 / \mathrm{Ln} \mathrm{N}(\mathrm{S}=$ número de especies de la muestra, $\mathrm{N}=$ número total de individuos en la muestra); el índice de diversidad de Shannon (H'n) (Shannon \& Weaver, 1949) definido como H'n $=\Sigma_{\mathrm{i}=1}^{\mathrm{s}} p_{i} \operatorname{Ln} p_{i}(\mathrm{pi}=$ probabilidad de presencia de la especie $i$ (número de individuos de la especie $i$ en la muestra dividida entre el número total de individuos en la muestra); y el índice de equidad de Pielou (J'n) (Pielou, 1966), definido como J'= H'/LnS.

Los datos fueron sometidos a prueba de normalidad (Shapiro-Wilk) y homoscedasticidad
(Levene) y presentaron una distribución normal y homogeneidad de varianza, por lo que no fue necesaria ninguna transformación. Se realizaron ANOVAs anidados para determinar las diferencias de los tres índices mencionados, así como del número de especies, entre el PNG y PNAX, entre sitios dentro de una misma AMP, y con respecto a la época climática. No fue necesario aplicar técnicas de rarefacción o acumulación de especies debido a que el esfuerzo fue el mismo.

Se realizó una prueba de $t$ de Student para comparar las medias de los índices de diversidad (H'y J') por sitio (PNG: Cuevas de Pedro, Encanto, Jardín de las Gorgonias y Yemayá; PNAX: Xahuayxol, Río Huach y Xcalak) teniendo en cuenta como factor la ausencia (PNG: 2003, 2004, 2007 y 2008; PNAX: 19971998, 2006 y 2007) y la presencia de pez león (PNG: 2013, 2014 y 2015; PNAX: 2010, 2014 y 2015). En el caso del PNAX se usaron curvas de rarefacción para analizar la diversidad debido a que el esfuerzo de muestreo fue diferente cada año en cada sitio (Xahuayxol, Río Huach y Xcalak). Para el análisis de los datos se utilizó el programa Statistica 8.0 (Statsoft, Inc.) y EstimateS (Colwell, 2009).

\section{RESULTADOS}

Comunidades de peces: Hubo diferencias significativas entre AMPs para el número de especies de peces $\left(\mathrm{F}_{1}=16.74, \mathrm{p}<0.001\right)$, pero no entre sitios de cada AMP (Fig. 2A). El PNG mostró mayores valores de riqueza que el PNAX (43.47 \pm 5.14 y $40.22 \pm 4.96$, respectivamente). El sitio de menor riqueza de las dos AMP fue Xcalak con 35 especies.

El índice de riqueza de Margalef mostró diferencias significativas entre ambas AMP $\left(\mathrm{F}_{1}=15.16, \mathrm{p}<0.001\right)$, mientras que los sitios de cada AMP no mostraron diferencias significativas entre ellos (Fig. 2B). El PNAX mostró mayores valores del índice de riqueza de Margalef (5.9 \pm 0.64$)$ que el PNG (5.6 \pm 0.79$)$. El índice de diversidad de Shannon manifestó diferencias significativas entre las dos AMP $\left(F_{1}=16.07, p<0.001\right)$ y entre los sitios de cada 

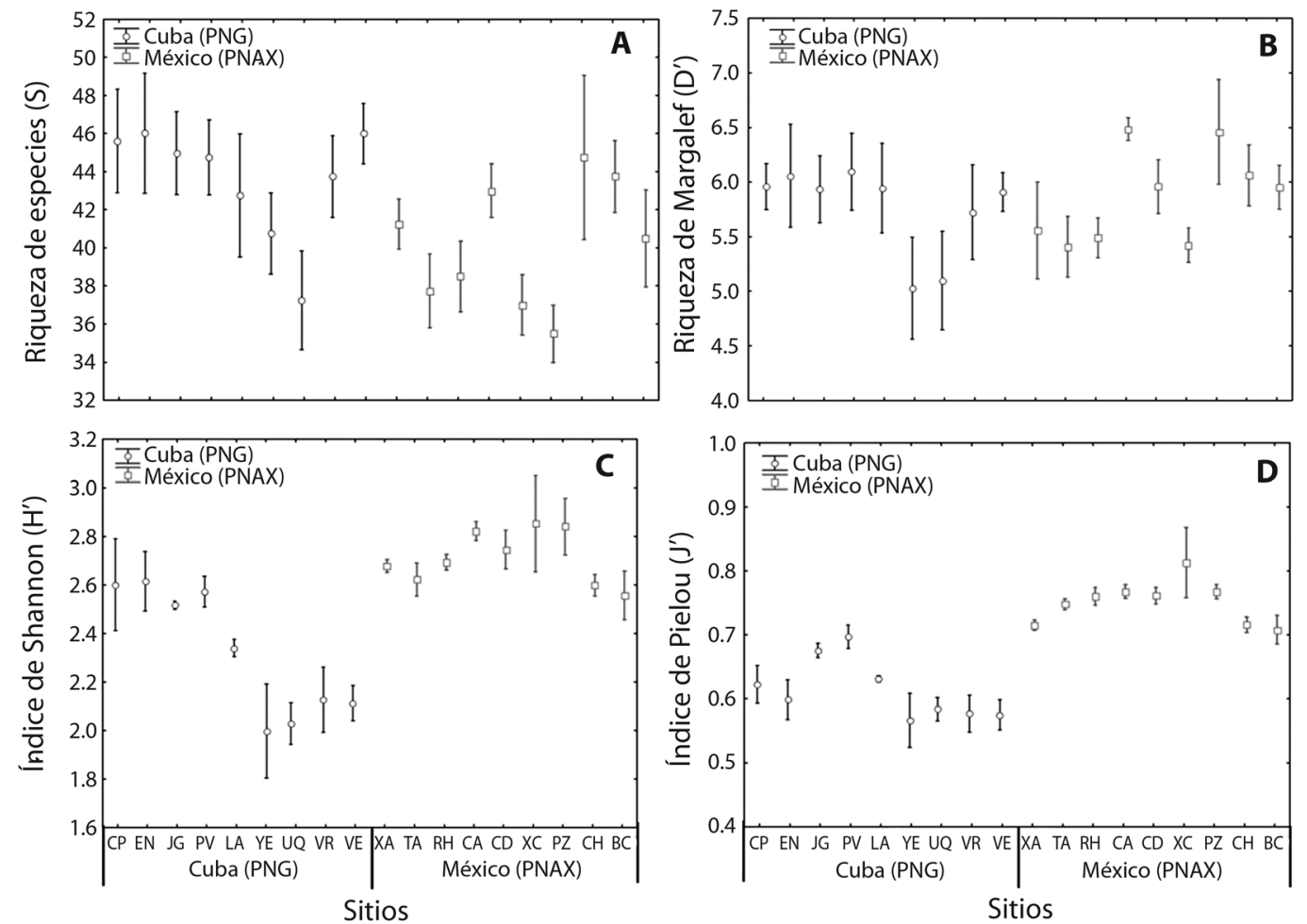

Fig. 2. Variación de índices de diversidad en los sitios del Parque Nacional Guanahacabibes (Cuba, PNG) y del Parque Nacional Arrecifes de Xcalak (México, PNAX). Número de especies (A), Índice de Margalef (B), Índice de diversidad de Shannon (C), Índice de equidad de Pielou (D). Las barras muestran la media \pm EE.

Fig. 2. Variation of diversity indexes in sampling sites of Guanahacabibes National Park (Cuba, PNG) and Xcalak Reef National Park (Mexico, PNAX). Number of species (A), Margalef Index (B), Shannon Diversity Index (C), Pielou Evenness Index (D). Bars show mean $\pm \mathrm{SE}$.

$\operatorname{AMP}\left(\mathrm{F}_{1}=31.70, \mathrm{p}<0.001\right)$ (Fig. 2C). Los valores de diversidad fueron mayores en el PNAX $(2.71 \pm 0.19)$ que en el PNG (2.34 \pm 0.31$)$. Los sitios de mayor diversidad en el PNAX fueron: La Poza, La Chimenea y Bacalar Chico, mientras, que en el PNG se destacó Cuevas de Pedro, Encanto y Patio de Vanesa. El índice de equidad de Pielou presentó un patrón similar, diferencias significativas entre las AMP $\left(\mathrm{F}_{1}=\right.$ 25.19, $\mathrm{p}<0.001)$ y entre los sitios de cada AMP $\left(\mathrm{F}_{1}=2.96, \mathrm{p}=0.001\right)$ (Fig. 2D). El PNAX mostró mayores valores $(0.75 \pm 0.05)$ que el PNG $(0.62 \pm 0.06)$.

Los análisis de varianza aplicados al número de especies y a los índices de Margalef, de equidad y de Shannon no mostraron diferencias significativas con respecto a la época climática dentro de cada AMP ni entre AMP (Fig. 3A-D). En los cuadros 1 y 2 se muestran los valores de los índices por sitio en cada AMP, para lluvia y seca.

En el PNG se registraron 152 especies de peces y en el PNAX 139, donde el PNG tuvo una media por sitio de 45 especies y el PNAX de 40 especies (Fig. 4 y 5). Las especies dominantes (Cuadro 1) fueron más abundantes en el PNG (Coryphopterus personatus/hyalinus, Clepticus parrae, Gramma loreto, G. melacara y Chromis multilineata) que, en el PNAX, donde las comunidades de peces fueron más 

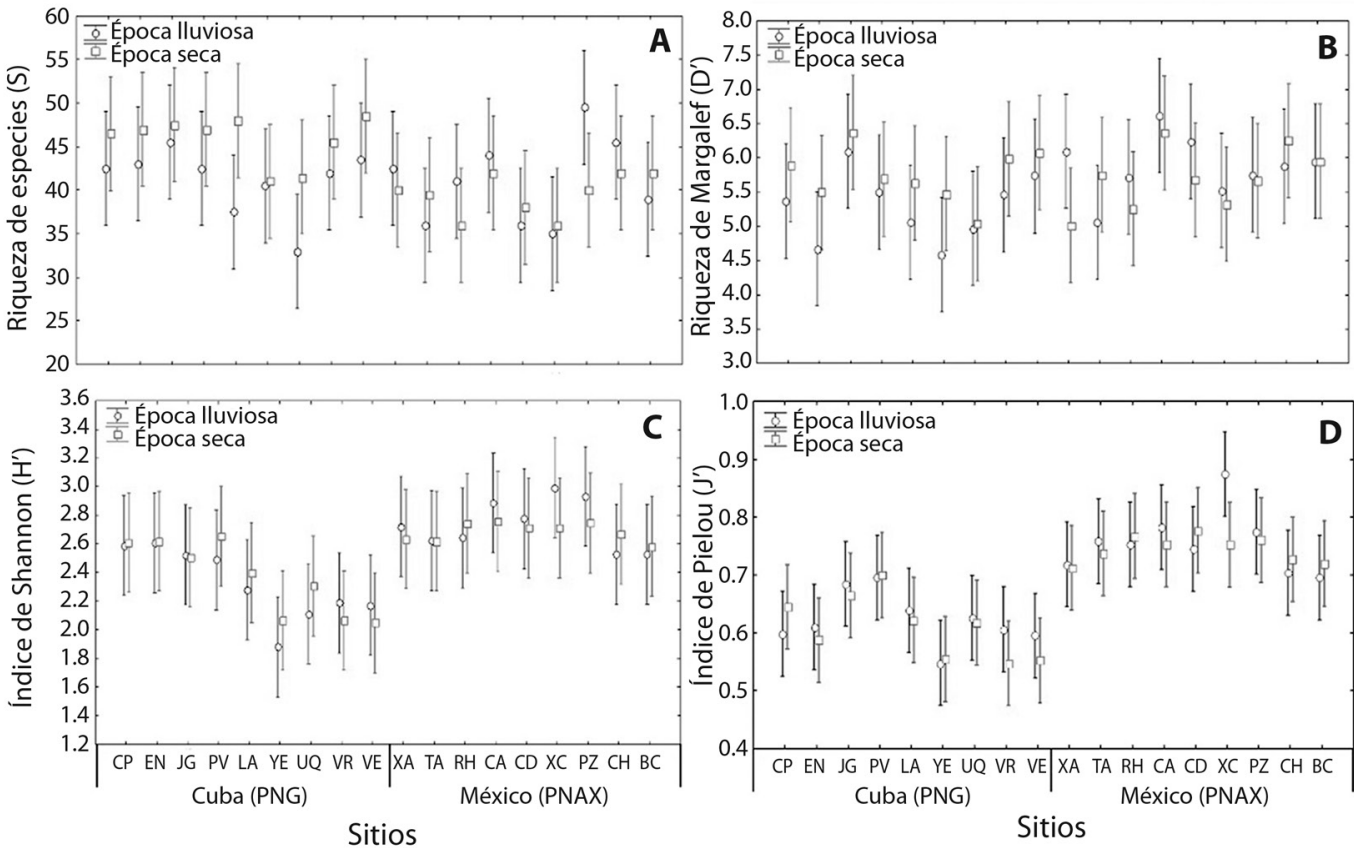

Fig. 3. Variación en los índices de diversidad en épocas de seca y lluvia por año en sitios del Parque Nacional Guanahacabibes (Cuba, PNG) y del Parque Nacional Arrecifes de Xcalak (México, PNAX). Número de especies (A), Índice de Margalef (B), Índice de equidad de Pielou (C), Índice de diversidad de Shannon(D). Las barras muestran la media \pm EE.

Fig. 3. Variation of diversity indexes in dry and rainy seasons in sampling sites per year of the Guanahacabibes National Park (Cuba PNG) and Xcalak Reef National Park (Mexico, PNAX). Number of species (A), Margalef Index (B), Pielou Evenness Index (C), Shannon Diversity Index (D). Bars show mean \pm ES.

\section{CUADRO 1}

Especies más abundantes en el Parque Nacional Guanahacabibes (Cuba) y Parque Nacional Arrecifes de Xcalak (México)

TABLE 1

Most abundant species in the Guanahacabibes National Park (Cuba) and Xcalak Reef National Park (Mexico)

\begin{tabular}{lcc}
\multicolumn{1}{c}{ Especies } & Abundancia \% (PNG) & Abundancia \% (PNAX) \\
Coryphopterus personatus/hyalinus & 27.49 & 2.14 \\
Clepticus parrae & 19.66 & 4.74 \\
Stegastes partitus & 10.18 & 11.8 \\
Chromis cyanea & 10.01 & 10.03 \\
Gramma loreto & 4.35 & 2.6 \\
Thalassoma bifasciatum & 4.32 & 14.23 \\
Chromis multilineata & 2.99 & 0.47 \\
Gramma melacara & 2.82 & 0 \\
Halichoeres garnoti & 1.39 & 4.35 \\
Ocyurus chrysurus & 1.15 & 1.04 \\
Canthigaster rostrata & 0.92 & 4.12 \\
Acanthurus coeruleus & 1.08 & 4.07 \\
Pterois volitans & 0.65 & 0.36 \\
Scarus taeniopterus & 0.61 & 3.52 \\
Abudefduf saxatilis & 0.04 & 3.3 \\
Scarus iseri & 0.42 & 2.99 \\
\hline
\end{tabular}




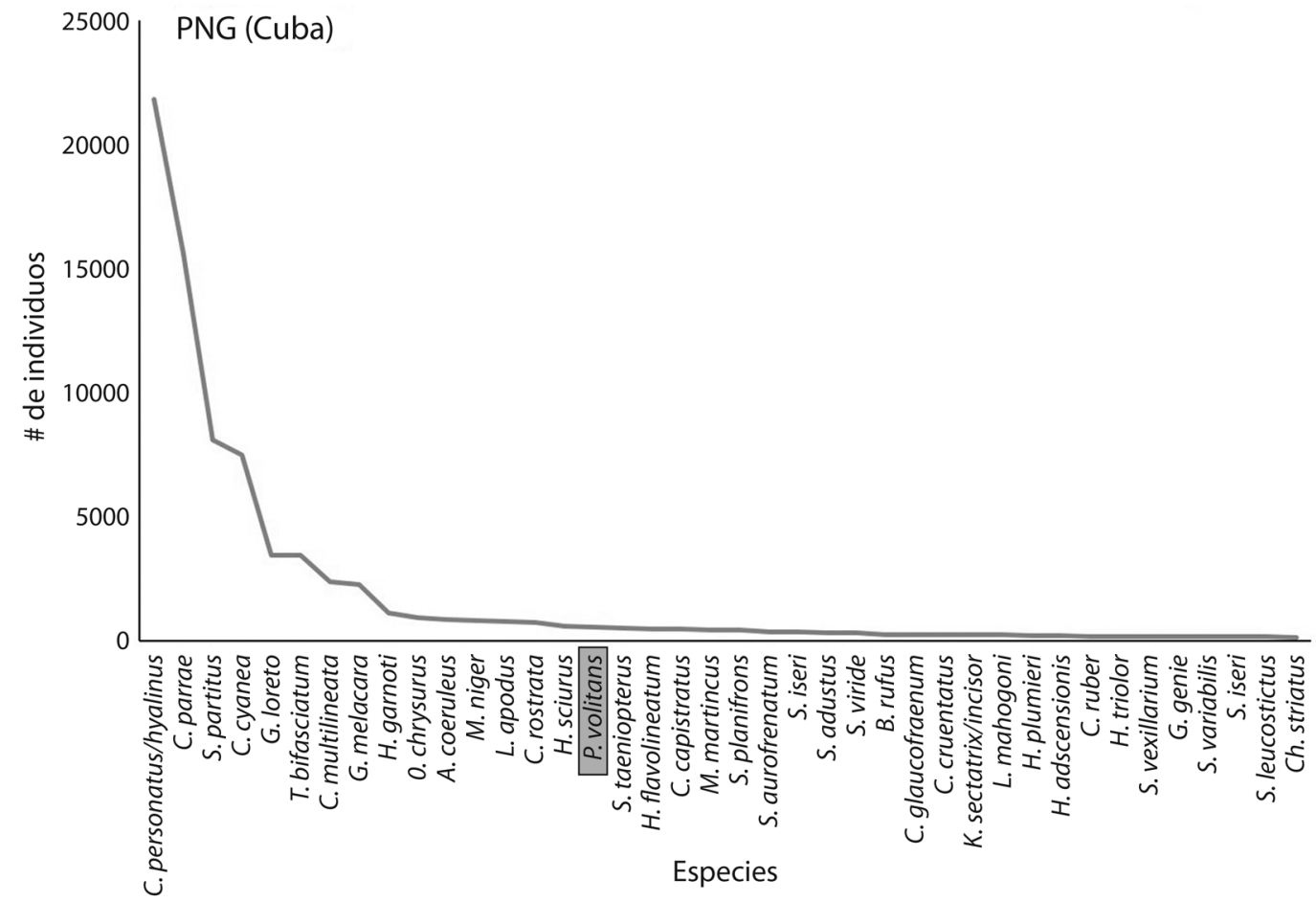

Fig. 4. Número de individuos de las 40 especies más abundantes en el Parque Nacional Guanahacabibes (PNG).

Fig. 4. Number of individuals of the 40 most abundant species in the Guanahacabibes National Park (PNG).

homogéneas. Destacó el pez león con 522 individuos en el PNG (8-31 ind/sitio) y sólo 114 individuos para el PNAX (0-8 ind/sitio).

Antes-después del pez león: Los índices de diversidad y equidad en función de la ausencia (2003-2008) y presencia (2009-2015) del pez león en el PNG no mostraron cambios en la mayoría de los sitios. Yemayá fue el único sitio que mostró diferencias significativas en el índice de diversidad de Shannon en función de la ausencia (H'media: $2.7 \pm 0.42$ ) o presencia (H'media: $1.97 \pm 0.38$ ) del pez león. El resto de los sitios presentaron valores similares de diversidad y equidad (Cuadro 2). En el PNAX, no se observaron diferencias significativas en estos índices en ningún sitio a partir de los resultados de la prueba de $t$ de Student (Cuadro 3). $\mathrm{Al}$ analizar la diversidad ponderada por curvas de rarefacción solo se encontraron diferencias significativas en Río Huach: la diversidad bajó de $3.11 \pm 0.08$ en $2006-07$ a $2.57 \pm 0.13$ en $2014-15(\mathrm{~F}=27.2 ; \mathrm{p}<0.05)$ y Xcalak disminuyó su diversidad de $3.3 \pm 0.08$ en 2006-07 a $2.9 \pm 0.07$ en $2014-15(\mathrm{~F}=9.80 ; \mathrm{p}<0.05)$.

\section{DISCUSIÓN}

En el PNG se registró menor número de especies de peces que los inventariados en otros estudios (Cobián et al., 2011), lo cual pudiera estar relacionado con el tipo de metodología utilizada. En esta área tradicionalmente se han realizado transectos lineales, los cuales se reconocen como muy efectivos para evaluar la diversidad, al cubrir un área relativamente grande y considerarse más útil para los organismos móviles y con distribución parchada (Claro \& García-Arteaga, 1994). El método estacionario es muy eficiente para determinar la frecuencia y abundancia relativa de los peces de manera rápida y con un mayor número de réplicas que le confieren alta confiabilidad estadística (Bohnsack \& Bannerot, 1986). En el 


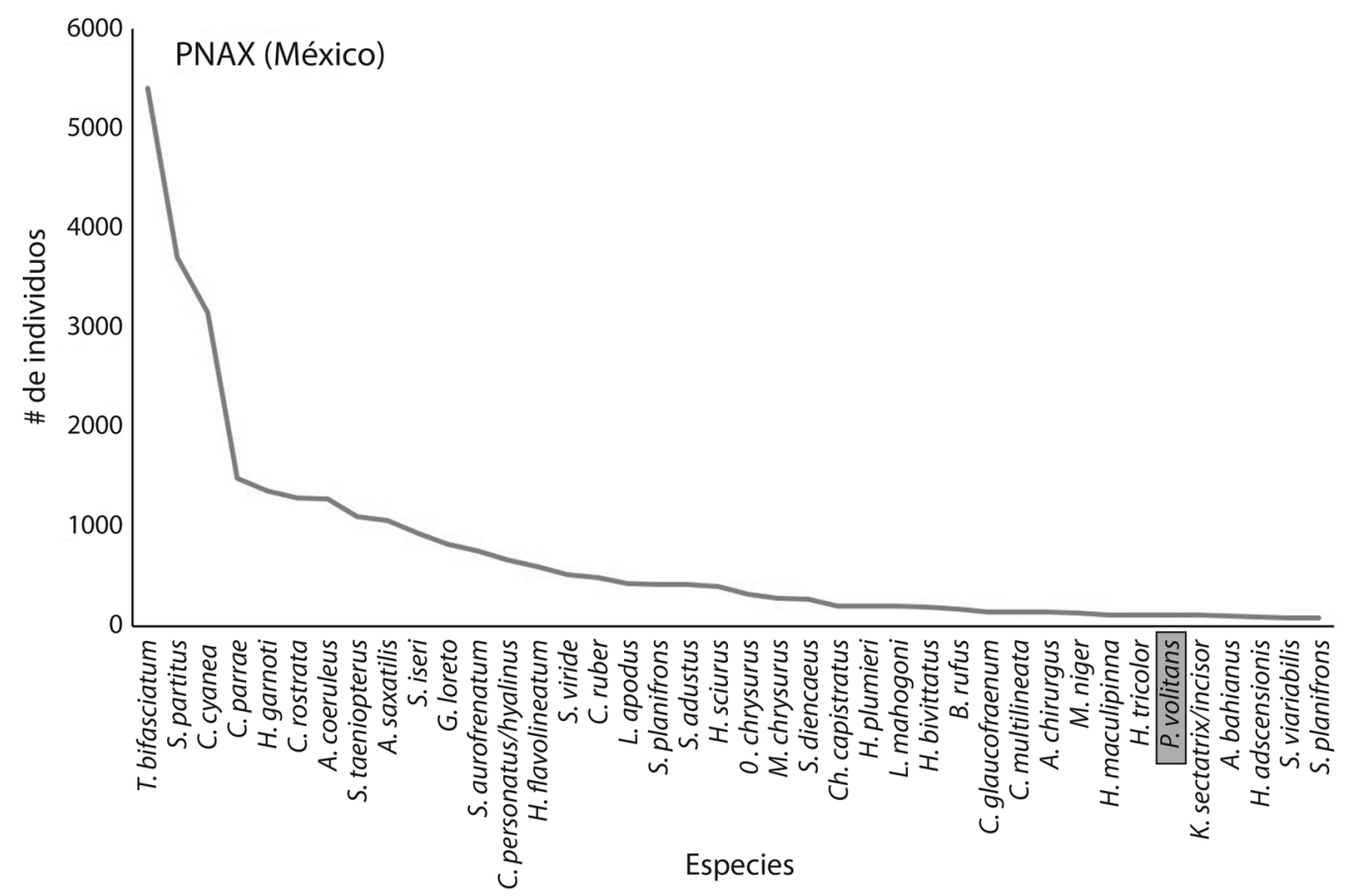

Fig. 5. Número de individuos de las 40 especies más abundantes en el Parque Nacional Arrecifes de Xcalak (PNAX).

Fig. 5. Number of individuals of the 40 most abundant species in the Xcalak Reef National Park (PNAX).

caso del PNAX se observaron 15 especies que no habían sido observadas en estudios anteriores con la misma metodología (Schmitter-Soto, Cruz-Martínez, Herrera, \& Hernández, 2007; Morales-Aranda et al., 2012). El biotopo de camellones y canales de la pendiente arrecifal del PNG presentó mayor riqueza debido a la elevada complejidad del relieve, que proporciona abundantes refugios y alimento para los peces; estos resultados coinciden con los encontrados por Cobián et al. (2011) en Cuba y con lo encontrado por otros autores en otras regiones (Alcolado, 1984; Núñez-Lara \& Arias-González, 1998).

Los sitios de mayor riqueza en el PNAX se ubicaron en el frente arrecifal, el hábitat de mayor complejidad. La Poza es considerada como un sitio especial debido a su estructura única (un cañón submarino) en el Caribe mexicano, por lo que el plan de manejo del área lo ubica dentro de sus principales objetos de conservación (CONANP, 2004). Por otra parte,
La Chimenea y Bacalar Chico se ubican en la zona de protección "Bacalar Chico" según la zonificación funcional del PNAX. En estos sitios está prohibida la pesca, solo se permite buceo contemplativo, investigación científica, monitoreo, restauración ecológica, protección y conservación ecológica. Esta zona limita con Belice, cuya zona está declarada como Parque Nacional y Reserva Marina Bacalar Chico (Grimshaw \& Paz, 2004), por lo que la zona sur del PNAX recibe los beneficios de ambas AMP, lo cual podría explicar la riqueza alta de especies. Estos sitios presentaron bajos valores de diversidad y equidad, debido a la dominancia de unas pocas especies.

Xcalak se ubica en la zona de aprovechamiento sustentable del PNAX, donde se permite buceo y pesca, por lo que es evidente la presión de pesca a las que están sometidas las comunidades de peces. Este arrecife manifiesta una degradación debido a la elevada presión de pesca ribereña para consumo y al comercio 
CUADRO 2

Variación del índice de diversidad de Shannon (H’n, en nats por individuo), índice de equidad de Pielou (J'n) y abundancia (media del número de individuos total de pez león) en cuatro sitios del Parque Nacional Guanahacabibes (CP: cuevas de Pedro, EN: Encanto, JG: Jardín de las Gorgonias y YE: Yemayá) antes y después de la presencia de pez león

TABLE 2

Variation of Shannon diversity index (in nats per individual), Pielou evenness index (J'n), and abundance (average number of lionfish) at four sites in Guanahacabibes National Park (CP: Cuevas de Pedro, EN: Encanto, JG: Jardín de las Gorgonias y YE: Yemayá) before and after the presence of lionfish

\begin{tabular}{|c|c|c|c|c|c|c|c|c|}
\hline Año & Épocas & Sitios & $\begin{array}{c}\text { Ausencia-presencia } \\
\text { de pez león }\end{array}$ & $\begin{array}{c}\text { Abundancia (Media } \\
\text { del número de individuos } \\
\text { total de pez león) }\end{array}$ & H’n & T-Student & J'n & T-Student \\
\hline 2003 & Lluvia & $\mathrm{CP}$ & Ausencia & 0.00 & 2.95 & \multirow{8}{*}{ NS } & 0.62 & \multirow{8}{*}{ NS } \\
\hline 2004 & Seca & $\mathrm{CP}$ & Ausencia & 0.00 & 3.40 & & 0.70 & \\
\hline 2007 & Lluvia & $\mathrm{CP}$ & Ausencia & 0.00 & 2.83 & & 0.69 & \\
\hline 2008 & Seca & $\mathrm{CP}$ & Ausencia & 0.00 & 2.55 & & 0.64 & \\
\hline 2013 & Lluvia & $\mathrm{CP}$ & Presencia & $2.05 \pm 1.35$ & 2.85 & & 0.56 & \\
\hline 2014 & Seca & $\mathrm{CP}$ & Presencia & $0.55 \pm 0.60$ & 2.23 & & 0.60 & \\
\hline 2014 & Lluvia & $\mathrm{CP}$ & Presencia & $0.65 \pm 0.74$ & 2.33 & & 0.64 & \\
\hline 2015 & Seca & $\mathrm{CP}$ & Presencia & $0.50 \pm 0.51$ & 2.99 & & 0.69 & \\
\hline 2003 & Lluvia & $\mathrm{EN}$ & Ausencia & 0.00 & 2.78 & \multirow{7}{*}{ NS } & 0.59 & \multirow{7}{*}{ NS } \\
\hline 2004 & Seca & $\mathrm{EN}$ & Ausencia & 0.00 & 3.39 & & 0.71 & \\
\hline 2007 & Lluvia & EN & Ausencia & 0.00 & 2.66 & & 0.67 & \\
\hline 2008 & Seca & $\mathrm{EN}$ & Ausencia & 0.00 & 2.74 & & 0.66 & \\
\hline 2013 & Lluvia & $\mathrm{EN}$ & Presencia & $0.40 \pm 0.58$ & 2.88 & & 0.57 & \\
\hline 2014 & Lluvia & $\mathrm{EN}$ & Presencia & $0.55 \pm 0.60$ & 2.34 & & 0.65 & \\
\hline 2015 & Seca & EN & Presencia & $0.65 \pm 0.81$ & 2.48 & & 0.65 & \\
\hline 2003 & Lluvia & JG & Ausencia & 0.00 & 3.14 & \multirow{8}{*}{ NS } & 0.65 & \multirow{8}{*}{ NS } \\
\hline 2004 & Seca & JG & Ausencia & 0.00 & 3.58 & & 0.73 & \\
\hline 2007 & Lluvia & JG & Ausencia & 0.00 & 2.65 & & 0.64 & \\
\hline 2008 & Seca & JG & Ausencia & 0.00 & 2.68 & & 0.68 & \\
\hline 2013 & Lluvia & JG & Presencia & $0.90 \pm 1.07$ & 2.55 & & 0.71 & \\
\hline 2014 & Seca & JG & Presencia & $0.80 \pm 1.05$ & 2.48 & & 0.68 & \\
\hline 2014 & Lluvia & JG & Presencia & $0.65 \pm 0.62$ & 2.50 & & 0.66 & \\
\hline 2015 & Seca & JG & Presencia & $0.50 \pm 0.60$ & 2.54 & & 0.65 & \\
\hline 2003 & Lluvia & YE & Ausencia & 0.00 & 2.79 & & 0.62 & \multirow{8}{*}{ NS } \\
\hline 2004 & Seca & YE & Ausencia & 0.00 & 3.25 & & 0.67 & \\
\hline 2007 & Lluvia & YE & Ausencia & 0.00 & 2.56 & & 0.58 & \\
\hline 2008 & Seca & YE & Ausencia & 0.00 & 2.23 & $\mathrm{t}=2.5326$ & 0.54 & \\
\hline 2013 & Lluvia & YE & Presencia & $1.55 \pm 1.90$ & 1.74 & $\mathrm{p}=0.0401$ & 0.52 & \\
\hline 2014 & Seca & YE & Presencia & $0.70 \pm 0.75$ & 1.63 & & 0.56 & \\
\hline 2014 & Lluvia & YE & Presencia & $0.70 \pm 0.80$ & 2.03 & & 0.58 & \\
\hline 2015 & Seca & YE & Presencia & $0.50 \pm 0.60$ & 2.50 & & 0.65 & \\
\hline
\end{tabular}

local, debido al incremento del turismo en la zona a pesar de ser un AMP (Garza-Pérez, 1999; Ruiz-Zárate, Hernández-Landa, González-Salas, Núñez-Lara, \& Arias-González, 2003). Otro sitio con similar presión de pesca es Siete Cocos Dentro, el cual presentó baja riqueza de especies, además de ubicarse en la laguna arrecifal, hábitat menos complejo; esto coincidió con lo encontrado en la mayoría de los estudios en esta área, mayor riqueza en el frente arrecifal que en la laguna arrecifal, la cual cuenta con grandes extensiones de 
CUADRO 3

Variación del índice de diversidad de Shannon (H’n, en nats por individuo), índice de equidad de Pielou (J'n) y abundancia (media del número de individuos total de pez león) en tres sitios del Parque Nacional Arrecifes de Xcalak (XA: Xahuayxol, RH: Río Huach, XC: Xcalak) antes y después de la presencia de pez león

TABLE 3

Variation of Shannon diversity index (H'n, in nats per individual), Pielou evenness index (J'n) and abundance (average number of lionfish) at three sites in Xcalak Reef National Park (XA: Xahuayxol, RH: Río Huach, XC: Xcalak) before and after the presence of lionfish

\begin{tabular}{|c|c|c|c|c|c|c|c|c|}
\hline Año & Épocas & Sitios & $\begin{array}{c}\text { Ausencia-presencia } \\
\text { de pez león }\end{array}$ & $\begin{array}{c}\text { Abundancia (Media del } \\
\text { número de individuos total } \\
\text { de pez león) }\end{array}$ & H’n & T-student & J'n & T-student \\
\hline $1997-1998$ & Seca & XA & Ausencia & 0.00 & 2.80 & \multirow{8}{*}{ NS } & 0.70 & \multirow{8}{*}{ NS } \\
\hline 1997-1998 & Lluvia & XA & Ausencia & 0.00 & 2.50 & & 0.60 & \\
\hline 2010 & Seca & XA & Ausencia & 0.00 & 2.70 & & 0.70 & \\
\hline 2010 & Lluvia & XA & Presencia & $0.04 \pm$ & 2.90 & & 0.70 & \\
\hline 2014 & Seca & XA & Presencia & $0.20 \pm 0.41$ & 2.64 & & 0.72 & \\
\hline 2014 & Lluvia & XA & Presencia & $0.25 \pm 0.55$ & 2.70 & & 0.70 & \\
\hline 2015 & Seca & XA & Presencia & $0.40 \pm 0.50$ & 2.63 & & 0.71 & \\
\hline 2015 & Lluvia & XA & Presencia & $0.35 \pm 0.80$ & 2.74 & & 0.74 & \\
\hline 1997 & Seca & RH & Ausencia & 0.00 & 2.87 & \multirow{8}{*}{ NS } & 0.76 & \multirow{8}{*}{ NS } \\
\hline 1998 & Lluvia & RH & Ausencia & 0.00 & 1.90 & & 0.50 & \\
\hline 2006 & Lluvia & RH & Ausencia & 0.00 & 3.20 & & 0.86 & \\
\hline 2014 & Seca & RH & Presencia & $0.10 \pm 0.30$ & 2.58 & & 0.74 & \\
\hline 2014 & Lluvia & RH & Presencia & $0.15 \pm 0.36$ & 2.46 & & 0.75 & \\
\hline 2015 & Seca & RH & Presencia & $0.35 \pm 0.48$ & 2.66 & & 0.76 & \\
\hline 2015 & Lluvia & RH & Presencia & $0.25 \pm 0.55$ & 2.79 & & 0.77 & \\
\hline 2006 & Lluvia & $\mathrm{XC}$ & Ausencia & 0.00 & 2.89 & & 0.80 & \\
\hline 2007 & Seca & $\mathrm{XC}$ & Ausencia & 0.00 & 2.57 & \multirow{7}{*}{ NS } & 0.70 & \multirow{7}{*}{ NS } \\
\hline 2010 & Seca & $\mathrm{XC}$ & Ausencia & 0.00 & 2.30 & & 0.60 & \\
\hline 2010 & Lluvia & $\mathrm{XC}$ & Ausencia & 0.00 & 2.80 & & 0.71 & \\
\hline 2014 & Seca & $\mathrm{XC}$ & Presencia & $0.30 \pm 0.47$ & 2.11 & & 0.70 & \\
\hline 2014 & Lluvia & $\mathrm{XC}$ & Presencia & 0.00 & 2.39 & & 0.68 & \\
\hline 2015 & Seca & $\mathrm{XC}$ & Presencia & 0.00 & 2.51 & & 0.74 & \\
\hline 2015 & Lluvia & $\mathrm{XC}$ & Presencia & $0.25 \pm 0.71$ & 2.10 & & 0.67 & \\
\hline
\end{tabular}

pastos marinos y arena (Carranza-Sánchez, Molina-lslas, Bezaury-Creel, López-Santos, \& McCann, 1994; Morales-Aranda et al., 2012). Esta tendencia ha sido encontrada en arrecifes con igual perfil en la región del Caribe mexicano (Núñez-Lara et al., 2003).

La mayoría de los sitios en PNG se ubican en la pendiente arrecifal (borde de pared). Este hábitat favorece una alta dominancia de unas pocas especies (Coryphopterus personatus/hyalinus, Gramma loreto, Chromis cyanea, C. multilineata, Stegastes partitus, Clepticus parrae), lo que hace que la equidad sea menor y, por lo tanto, el índice de diversidad también. Estas especies conformaron el $73 \%$ de todos los individuos en un estudio similar por Cobián y Chevalier Monteagudo (2009) en la misma área. La misma tendencia fue observada en la pendiente arrecifal de Bahía de Cochinos (Cuba) por Chevalier y Cárdenas (2005).

Yemayá y Uvero Quemado fueron los sitios menos diversos en el PNG, lo que pudiera estar relacionado con pesca ilegal en esta zona (aunque no intensa), que se encuentra más desprotegida y accesible a los pescadores. La baja equidad se debe a la dominancia de pocas 
especies, fundamentalmente C. cyanea. C. multilineata y $C$. parrae que habitan en la columna de agua, lo que coincide con estudios anteriores (Cobián et al., 2011).

La época climática no tuvo efectos sobre los índices de diversidad. Estos resultados coinciden con los encontrados por Cobián et al. (2011) en los mismos sitios. En el PNAX, tampoco se observaron diferencias en cuanto a las épocas en este estudio. Sin embargo, Schmitter-Soto et al. (2007) encontraron diferencias significativas para el índice de riqueza, específicamente en el sitio Xahuayxol. Con respecto al índice de equidad según SchmitterSoto et al. (2007), las especies muestreadas en las distintas épocas climáticas para 2010 tuvieron mayor homogeneidad en distribución que en 2000 (Morales-Aranda et al., 2012). Estos autores mencionan que las diferencias estacionales podrían estar relacionadas con la pesca histórica en el área.

El pez león se ubicó entre las especies más abundantes en los arrecifes coralinos del PNG, ocupando el lugar 16 en la lista a solo seis años de su primer registro. En el PNG no existe un programa de manejo dirigido para el pez león, solo se han realizado capturas con fines científicos (Cobián et al., 2016). En cambio, en el PNAX se ejecuta un programa eficiente de manejo desde el 2009 a través, de torneos de pesca y programas gubernamentales de empleo temporal (Sabido-Itzá, Medina, Navarrete, Gómez-Poot, \& García-Rivas, 2016). En el PNAX el pez león ocupó el lugar 35 y fue menos abundante, lo que pudiera indicar el efecto positivo de los programas sistemáticos de remoción (Barbour, Allen, Frazer, \& Sherman, 2011; De León et al., 2013). No obstante, en el PNG existen otros factores ambientales $\mathrm{y}$ físicos que favorecen la elevada abundancia de pez león, como una mayor complejidad estructural del arrecife (superior a la observada en el PNAX), que provee abundante refugio y alimento a esta especie (Cobián et al., 2016), también pudieran influir la geomorfología de la zona, la dinámica de las corrientes u otras variables abióticas como la temperatura, que sería interesante analizar en otros estudios.
La mayoría de los sitios en el PNG no mostraron cambios en los índices, al comparar datos antes y después de la invasión de pez león. Sin embargo, Yemayá mostró una disminución significativa en el índice de diversidad (bajos valores riqueza, diversidad y equidad), lo que se ha relacionado con la pesca furtiva del área. Yemayá presentó un incremento de pez león de manera significativa del 2009-2013, (Cobián et al., 2016) donde se mantienen las elevadas abundancias de pez león $(0.086 \pm 1.20)$. En este caso se aplicó la metodología de conteos estacionarios a una escala menor, mientras, que Cobián et al. (2016) aplicaron transectos lineales, abarcando una mayor área. También se consideraron abundantes los mesodepredadores nativos, Cephalopholis cruentata ( 0.37 $\pm 0.055)$, Lutjanus apodus $(0.075 \pm 3.21)$ y $C$. fulva $(0.010 \pm 0.30)$, los cuales no son objeto de pesca en la zona. Según evidencias de estudios anteriores, los efectos negativos en las comunidades de peces podrían ser mayores cuando estén presentes los depredadores nativos y el pez león (Albins, 2013), lo cual se uniría a los efectos de extracción existentes del pez león. Estos efectos no fueron detectables a seis años de la invasión en el PNG, por lo que se sugiere continuar con los monitoreos en esta área, con diseños que puedan detectar estos impactos a largo plazo, ya que el pez león pudiera desestabilizar la dinámica depredador-presas nativas, donde el invasor puede causar tres veces una mortalidad mayor que los mesodepredadores nativos (Ingeman \& Webster, 2015).

Rio Huach y Xcalak reportaron bajas abundancias de pez león durante este estudio (Río Huach: 0.02 \pm 0.35 ; Xcalak: 0.013 \pm 0.44 ), lo cual pudiera deberse al programa de captura de pez león que organiza el PNAX desde 2009 a través de los programas de empleo temporal. Por lo anterior, asumimos que los cambios en la diversidad detectados se deben fundamentalmente a la pesca histórica del área (SchmitterSoto et al., 2007; Morales-Aranda et al., 2012).

Las diferencias encontradas en cuanto a riqueza, diversidad y equidad en las comunidades de peces en ambas AMP no parecen estar asociadas a la abundancia del pez león 
y sí a la estructura particular de los arrecifes y a los efectos de la pesca. En arrecifes de Venezuela, Elise, Urbina-Barreto, Boadas-Gil, Galindo-Vivas y Kulbicki (2015) no detectaron cambios significativos en la estructura de las comunidades de peces nativos al analizar los efectos del pez león. Sin embargo, Green et al. (2012), Côté et al. (2013), Albins (2013), Albins, (2015) y Dahl, Patterson III y Snyder (2016) mencionan que el pez león ha causado disminuciones rápidas y significativas en la riqueza y diversidad de especies en los arrecifes. Sugerimos continuar los monitoreos en las AMP de Cuba y México, así como la implementación de experimentos que permitan revelar el efecto real del pez león sobre las especies presentes para detectar posibles cambios en la estructura de las comunidades de peces nativos. También, recomendamos trazar estrategias de control dirigidas al pez león: torneos de pesca, colocación de trampas y redes en zonas profundas, motivar su consumo o el uso en la artesanía. Algunas de estas acciones, como la promoción de consumo y torneos de pesca, han sido efectivas en otros sitios del Caribe (Morris \& Akins, 2009). La implementación adecuada de estas estrategias puede contribuir a la conservación de las especies nativas en los arrecifes del PNG y PNAX.

\section{AGRADECIMIENTOS}

Agradecemos a los especialistas de los parques nacionales Guanahacabibes y Arrecifes de Xcalak, al personal del Complejo Marinas Gaviota María la Gorda-Cabo de San Antonio y al Proyecto Archipiélagos de Sur GEF/PNUD (Cuba) por el apoyo de la logística durante los muestreos. A Gabriel Cruz-Ruiz por la elaboración del mapa. A Idea Wild por la donación de equipos para el análisis y procesamiento de datos y muestras. El artículo forma parte de la tesis doctoral de la primera autora, quien recibió una beca del Consejo Nacional de Ciencia y Tecnología de México.

\section{RESUMEN}

El pez león (Pterois volitans) invadió la región del Caribe y tiene el potencial de alterar la composición y estructura de las comunidades de peces en los arrecifes coralinos. El objetivo de este estudio fue analizar los índices de diversidad en las comunidades de peces nativos en sitios invadidos por el pez león en dos áreas marinas protegidas (AMP) del Caribe y compararlos con datos previos a la invasión. En ambas AMP, Parque Nacional Guanahacabibes (PNG) en el occidente de Cuba y Parque Nacional Arrecifes de Xcalak (PNAX) en el S de Quintana Roo, se realizaron censos visuales de las especies de peces en hábitats durante las épocas de seca y lluvia del 2013-2015. Se evaluaron nueve sitios, mediante conteos estacionarios. Se registró mayor riqueza de especies en el PNG (43.47 \pm 5.14$)$ que en el PNAX (40.22 \pm 4.96$)$. No se observaron diferencias entre épocas en ninguna de las AMP. El pez león se ubicó entre las especies más abundantes del PNG. La abundancia media en el PNG $(0.76 \pm 1.25)$ fue mayor a la registrada en el PNAX $(0.19 \pm 0.46)$. La diversidad disminuyó después de la llegada del pez león en un solo sitio del PNG y en dos sitios del PNAX, pero al parecer estos resultados están más asociados al efecto de la pesca que a la presencia del pez león. A partir de los resultados y asumiendo que los cambios en las comunidades de peces por el pez león podrían no detectarse aún, recomendamos seguir los monitoreos de los descriptores comunitarios para detectar cambios futuros en las comunidades de peces.

Palabras clave: diversidad, especies invasoras, peces marinos, arrecife de coral, efectos de depredadores.

\section{REFERENCIAS}

Albins, M. A. (2013). Effects of invasive Pacific red lionfish Pterois volitans versus a native predator on Bahamian coral-reef fish communities. Biological Invasions, 15(1), 29-43. DOI: 10.1007/s10530-012-0266-1

Albins, M. A. (2015). Invasive Pacific lionfish Pterois volitans reduce abundance and species richness of native Bahamian coral-reef fishes. Marine Ecology Progress Series, 522, 231-243. DOI: 10.3354/meps11159

Alcolado, P. M. (1984). Utilidad de algunos índices ecológicos estructurales en el estudio de comunidades marinas de Cuba. Ciencias Biológicas, 11, 61-77.

Alcolado, P. M. (1998). Conceptos e índices relacionados con la diversidad. Avicennia, 8(9), 7-21.

Ballew, N. G., Bacheler, N. M., Kellison, G. T., \& Schueller, A. M. (2016). Invasive lionfish reduce native fish abundance on a regional scale. Scientific Reports, 6 . DOI: $10.1038 /$ srep32169 
Barbour, A. B., Allen, M. S., Frazer, T. K., \& Sherman, K. D. (2011). Evaluating the potential efficacy of invasive lionfish (Pterois volitans) removals. PloS One, 6(5), e19666. DOI: 10.1371/journal.pone.0019666

Bohnsack, J. A., \& Bannerot, S. P. (1986). A stationary visual census technique for quantitatively assessing community structure of coral reef fishes. NOAA Technical Report NMFS, 41, 1-15.

Brock, V. J. (1954). A preliminary report on a method of estimating reef fish populations. The Journal of Wildlie Management, 18, 297-308. DOI: 10.2307/3797016

Capote López, R. P., Mitrani Arenal, I., \& Suárez, A. G. (2012). Conservación de la biodiversidad cubana y cambio climático en el archipiélago cubano. Anales de la Academia de Ciencias de Cuba, 1, 1-25.

Carranza-Sánchez, J., Molina-lslas, C., Bezaury-Creel, J. E., López-Santos, C., \& McCann, J. (1994). Caracterización de la zona de Xcalak. Quintana Roo. México. Propuesta para el establecimiento del Parque Nacional “Arrecifes de Xcalak". Sian Ka'an Serie Documentos, 5, 1-68.

Chevalier, P. P., \& Cárdenas, A. L. (2005). Variación espacial y temporal de las asociaciones de peces en arrecifes costeros de la costa oriental de la Bahía de Cochinos. I: Abundancia y Diversidad. Revista de Investigaciones Marinas, 26(1), 45-57.

Chevalier, P. P., Gutiérrez, E., Ibarzábal, D., Romero, S., Isla, V., Calderín, J., \& Hernández, E. (2008). Primer registro de Pterois volitans (Pisces: Scorpaenidae) para aguas cubanas. Solenodon, 7, 37-40.

Claro, R., \& García-Arteaga, J. P. (1994). Estructura de las comunidades de peces en los arrecifes del grupo insular Sabana-Camagüey, Cuba. Avicennia, 2, 83-107.

Cobián, D., \& Chevalier Monteagudo, P. P. (2009). Evaluación de las asociaciones de peces de los arrecifes coralinos del Centro Internacional de Buceo María la Gorda. Parque Nacional Guanahacabibes. Cuba. Revista Ciencias Marinas y Costeras, 1, 111-125.

Cobián, D., Claro, R., Chevalier, P. C., Perera, S., \& Caballero, H. (2011). Estructura de las asociaciones de peces en los arrecifes coralinos del Parque Nacional Guanahacabibes. Cuba. Revista Ciencias Marinas y Costeras, 3,153-169.

Cobián-Rojas, D., Chevalier, P., Schmitter-Soto, J. J., Wong, R. I. C., Torres, H. S., Sansón, E. C., ..., \& Perera-Valderrama, S. (2016). Density, size, biomass, and diet of lionfish in Guanahacabibes National Park, western Cuba. Aquatic Biology, 24(3), 219-226. DOI: 10.3354/ab00651

Colwell, R. K. (2006). EstimateS: statistical estimation of species richness and shared species from simples, (version 8.0.). Retrieved from http://purl.oclc. org/estimates
Côté, I. M., Green, S. J., \& Hixon, M. A. (2013). Predatory fish invaders: insights from Indo-Pacific lionfish in the western Atlantic and Caribbean. Biological Conservation, 164, 50-61. DOI: 10.3354/meps09992

Comisión Nacional de Áreas Naturales Protegidas (CONANP). (2004). Programa de Manejo Parque Nacional, Arrecifes de Xcalak., México. DOI: 10.1017/CBO9781107415324.004

Dahl, K. A., Patterson III, W. F., \& Snyder, R. A. (2016). Experimental assessment of lionfish removals to mitigate reef fish community shifts on northern Gulf of Mexico artificial reefs. Marine Ecology Progress Series, 558, 207-221. DOI: 10.1371/journal. pone. 0105852

De León, R., Vane, K., Bertuol, P., Chamberland, V. C., Simal, F., Imms, E., \& Vermeij, M. J. (2013). Effectiveness of lionfish removal efforts in the southern Caribbean. Endangered Species Research,22(2), 175182. DOI: $10.3354 /$ esr00542

Elise, S., Urbina-Barreto, I., Boadas-Gil, H., GalindoVivas, M., \& Kulbicki, M. (2015). No detectable effect of lionfish (Pterois volitans and P. miles) invasion on a healthy reef fish assemblage in Archipelago Los Roques National Park, Venezuela. Marine Biology, 162, 319-330. DOI: 10.1007/s00227-014-2571-y

Garza-Pérez, R. (1999). Análisis comparativo de cuatro comunidades coralinas arrecifales del Caribe mexicano (Tesis de Maestría). Centro de Investigación y de Estudios Avanzados del Instituto Politécnico Nacional, Mérida. Yucatán.

Gell, F. R., \& Roberts, C. M. (2002). The fishery effects of marine reserves and fishery closure. Washington DC: WWF-US.

Gell, F. R., \& Roberts, C. M. (2003). Benefits beyond boundaries: the fishery effects of marine reserves. Trends in Ecology \& Evolution, 18(9), 448-455. DOI: 10.1016/S0169-5347(03)00189-7

González-Ferrer, S., Caballero Aragón, H., Alcolado, P. M., Jiménez, A., Martín, F., \& Cobián, D. (2007). Diversidad de corales pétreos en once sitios de buceo recreativo de "María la Gorda", Cuba. Revista de Investigaciones Marinas, 28(2), 121-130.

Green, S. J., Akins, J. L., Maljković, A., \& Côté, I. M. (2012). Invasive lionfish drive Atlantic coral reef fish declines. PloS One, 7(3), e32596. DOI: 10.1371/ journal.pone.0032596

Grimshaw, T., \& Paz, G. (2004). The Revised Bacalar Chico National Park and Marine Reserve Management Plan. San Pedro, Belice: Green Reef Environmental Institute.

Gutiérrez, F. (2006). Estado de conocimiento de especies invasoras. Propuesta de lineamientos para el control 
de los impactos. Bogotá: Instituto de Investigación de Recursos Biológicos Alexander von Humboldt.

Heller, N. E., \& Zavaleta, E. S. (2009). Biodiversity management in the face of climate change: a review of 22 years of recommendations. Biological Conservation, 142(1), 14-32. DOI: 10.1016/j.biocon.2008.10.006

Hixon, M. A., Green, S. J., Albins, M. A., Akins, J. L., \& Morris Jr, J. A. (2016). Lionfish: a major marine invasion. Marine Ecology Progress Series, 558, 161-165. DOI: $10.3354 /$ meps 11909

Hughes, T. P., Baird, A. H., Bellwood, D. R., Card, M., Connolly, S. R., Folke, C., \& Lough, J. M. (2003). Climate change, human impacts, and the resilience of coral reefs. Science, 301(5635), 929-933. DOI: $10.1126 /$ science. 1085046

Ingeman, K. E., \& Webster, M. S. (2015). Native prey mortality increases but remains density-dependent following lionfish invasion. Marine Ecology Progress Series, 531, 241-252. DOI: 10.3354/meps11332

Magurran, A. E. (2004). Measuring Biological Diversity. Nueva York: John Wiley \& Sons.

Margalef, R. (1951). Diversidad de especies en las comunidades naturales. Publicaciones de. Instituto de Biología Aplicada de Barcelona, 9, 5-27.

Mellin, C., MacNeil, M. A., Cheal, A. J., Emslie, M. J., \& Caley, M. J. (2016). Marine protected areas increase resilience among coral reef communities. Ecology Letters, 19(6), 629-637. DOI: 10.1111/ele.12598

Mendoza, R., Luna, S., \& Aguilera, C. (2015). Risk assessment of the ornamental fish trade in Mexico: analysis of freshwater species and effectiveness of the FISK (Fish Invasiveness Screening Kit). Biological Invasions, 17, 3491-3502.

Morales-Aranda, A. A., Schmitter-Soto, J. J., \& Herrera Pavón, R. L. (2012). Evaluación de un área marina protegida en el Caribe: Un análisis antes-después/ control-impacto con peces arrecifales. In Del Moral Flores, L. F., Martínez Pérez, J. A., Franco López, J., Ramírez Villalobos, J., \& Tello Musi, J. L. (Eds.), Investigación Ictiológica en México. Temas selectos en honor al Dr. José Luis Castro Aguirre (pp. 231-245). Ciudad de México: Universidad Nacional Autónoma de México.

Morris, J. A., Jr., \& Akins, J. L. (2009). Feeding ecology of invasive lionfish (Pterois volitans) in the Bahamian archipelago. Environmental Biology of Fishes, 86, 389-398.

Mouillot, D., Parravicini, V., Bellwood, D. R., Leprieur, F., Huang, D., Cowman, P. F., ..., \& Guilhaumon, F. (2016). Global marine protected areas do not secure the evolutionary history of tropical corals and fishes. Nature Communications, 7, 10359. DOI: 10.1038/ ncomms 10359
Núñez-Lara, E., \& Arias-González, E. (1998). The relationship between reef fish community structure and environmental variables in the southern Mexican Caribbean. Journal of Fish Biology, 53(sA), 209-221. DOI: $10.1111 / \mathrm{j} .1095-8649$. 1998.tb01028.x

Núñez-Lara, E., González-Salas, C., Ruiz-Zárate, M. A., Hernández-Landa, R., \& Arias-González, E. J. (2003). Condition of coral reef ecosystems in centralsouthern Quintana Roo (Part 2: Reef fish communities). Atoll Research Bulletin, 496, 338-359.

Palumbi, S. R. (2004). Marine reserves and ocean neighborhoods: the spatial scale of marine populations and their management. Annual Review of Environment and Resources, 29, 31-68. DOI: 0.1146/annurev. energy.29.062403.102254

Pecenko, Y. A. (1982). Principios y métodos de análisis cuantitativo en investigaciones faunísticas. Moscú: Editorial Nauka.

Peet, R. K. (1974). The measurement of species diversity. Annual Review of Ecology and Systematics, 285-307.

Pielou, E. C. (1966). The measurement of diversity in different types of biological collections. Journal of Theoretical Biology, 13, 13- 144. DOI: 10.1016/0022-5193(66)90013-0

Rands, M. R., Adams, W. M., Bennun, L., Butchart, S. H., Clements, A., Coomes, D., ..., \& Sutherland, W. J. (2010). Biodiversity conservation: challenges beyond 2010. Science, 329(5997), 1298-1303. DOI: 10.1126/ science. 1189138

Rodríguez-Zaragoza, F. A., Cupul-Magaña, A. L., GalvánVilla, C. M., Ríos-Jara, E., Ortiz, M., Robles-Jarero, E. G., ..., \& Arias-González, J. E. (2011). Additive partitioning of reef fish diversity variation: a promising marine biodiversity management tool. Biodiversity and Conservation, 20(8), 1655-1675. DOI: 10.1007/s10531-011-0053-9

Ruiz-Zárate, M. A., Hernández-Landa, R. C., GonzálezSalas, C., Núñez-Lara, E., \& Arias-González, J. E. (2003). Condition of Coral Reefs Ecosystems in Central-southern Quintana Roo. Mexico (Part 1: Stony corals and algae). Atoll Research Bulletin, 496, 318-337.

Russ, G. R., \& Alcala, A. C. (2004). Marine reserves: long-term protection is required for full recovery of predatory fish populations. Oecologia, 138(4), 622627. DOI: $10.1007 / \mathrm{s} 00442-003-1456-4$

Sabido-Itzá, M. M., Gómez Poot, J. M., Medina Quej, A., García Rivas, M. C., \& Hadad López, W. (2012). Dinámica poblacional del pez león (Pterois volitans) en el Parque Nacional Arrecifes de Xcalak (PNAX), Quintana Roo, Caribe mexicano. Proceedings of the 64th Gulf and Caribbean Fisheries Institute, 64, 67-74. 
Sabido-Itzá, M. M, Medina-Quej, A., de Jesús-Navarrete, A., Gómez-Poot, J. M., \& García-Rivas, M. C. (2016). Uso de la estructura de tallas como evidencia del establecimiento poblacional del pez león Pterois volitans (Scorpaeniformes: Scorpaenidae) en el sur del Caribe Mexicano. Revista de Biología Tropical, 64(1), 36953-37862. DOI: 10.15517/rbt.v64i1.18943

Santos, T., \& Tellería, J. L. (2006). Pérdida y fragmentación del hábitat: efecto sobre la conservación de las especies. Ecosistemas, 15(2), 3-12.

Shannon, C. E., \& Weaver, W. (1949). The mathematical theory of communication. Urbana: University of Illinois Press.

Schmitter-Soto, J. J., Aguilar-Perera, A., Avilés Torres, S., Herrera, R., Caballero, J. A., \& Carvajal, N. (1998). Distribución y abundancia de la ictiofauna de la iciofauna arrecifal en la costa sur de Quintana Roo. Informe Final. Chetumal, México: CONACyT/ECOSUR.
Schmitter-Soto, J. J., Cruz-Martínez, A., Herrera, R., \& Hernández, A. (2007). Los peces de la costa sur de Quintana Roo: una década de cambios. Informe Final Chetumal, México: Fondo para el Sistema Arrecifal Mesoamericano y Fondo Mexicano para la Conservación de la Naturaleza.

StatSoft, Inc. (Version 6) [Data analysis software system]. Tulsa, USA: StatSoft, Inc.

Willis, T. J. (2003). Protection of exploited fishes in temperate regions: high density and biomass of snapper Pagrus auratus (Sparidae) in northern New Zealand marine reserve. Journal of Applied Ecology, 40, 214227. DOI: $10.1046 /$ j.1365-2664.2003.00775.x

Wong, C. P., Jiang, B., Kinzig, A. P., Lee, K. N., \& Ouyang, Z. (2015). Linking ecosystem characteristics to final ecosystem services for public policy. Ecology Letters, 18(1), 108-118. 